\title{
Quality Specification, Testing and Certification of Bespoken, Open Source and Commercial Off-The-Shelf Systems
}

\author{
Hans-Ludwig Hausen \\ FRAUNHOFER \\ Schloss Birlinghoven, D-53754 St. Augustin, Germany \\ Hans-Ludwig_Hausen@_fit.fraunhofer.de
}

\begin{abstract}
The seminar will cover the principles as well as the best practices of software system quality assurance (comprising inspection, verification, validation, black and white box test, measurement and assessment, and the normative quality characteristics) for procedural, object-oriented, aspectoriented and agent-based dependable software. Attendees will exercise proven techniques for goal-directed quality specification, testing, measurement, scaling and assessment for software certification. Assessment of both the software product as well as the software process will be discussed with respect to its relevance for such acceptance assessments. A standardized process model for measurement, assessment and certification of dependable software will be used to make the attendees familiar with this comprehensive assessment procedure and to learn how to embed it into today's standardized or non-standardized software processes..
\end{abstract}

What is software quality and what is quality specification, evaluation, assessment and certification? Why do we need it? Well, we ask for software quality specification, assessment and certification because we want to be sure that the product we want to apply provides the expected service correctly with respect to both functional and non-functional requirements. If we are assessing software we check whether the actual service we can get from the present version of the product is (at least to some degree) equivalent to the required service. We assume that the actual service is provided by a program that has been coded under several conditions and constrains and thus not being a one-to-one translation of the required service. Complementary we introduce a third layer in our approach called specified service, where we define what has to be done on the computer. The actual service might be considered as the layer describing how the service is to be accomplished. Why and what for the service is needed is already defined in the required service layer.

Please use the following format when ciling this chapler:

Hausen, H.-L., 2007, in IFIP International Federation for Information Processing, Volume 234, Open Source Development, Adoption and Innovation, eds. J. Feller, Fitzgerald, B., Scacchi, W., Sillitti, A., (Boston: Springer), pp. 365-366. 
In such a layered product environment assessment is performed using assessment methods such as inspection, testing, verification and measurement to check the actual service against the specified service and the expected service. These assessment methods have to be supported by appropriate tools. For the assessment we also need to know which characteristics of the product have to be considered and what is the threshold for them. In order to be able (i.e. "to be allowed") to certify a product, i.e. to put a quality seal on the product, we have to evaluate assess all product layers with the required characteristics using appropriate methods and tools on both product documents and process documents. As a consequence we have to handle product, process, characteristics, methods and tools as wells as their interaction in a defined, coherent procedure.

For effective quality specification, testing and certification the product and process elements have to be identified and evaluated with respect to selected, required characteristics. Appropriate methods and tools have to be applied to the product and process documents to check those characteristics. The essential problem domains are: software product, software process, software characteristics, software methods and software tools for procedural, object-oriented or agent-based dependable software systems. Proven techniques for goal-directed quality specification, measurement, scaling and assessment are mandatory. Obviously one has also to consider norms, regulations or standards such as the ones for

- Software Quality Specification and Evaluation: ISO9126 and ISO 14598

- Evaluators Guide according ISO9126 and ISO 14598

- COTS Quality Specification and Evaluation: ISO 12119

- COTS Evaluation Guide according ISO 12119

- ISO 25000 series

And finally, a standardized process model for measurement, assessment and certification of dependable software is required applicable in the context of today's standardized as well as within non-standardized software processes.

\section{References}

1 Software Evaluation for Certification; Andrew Rae, P. Robert, Hans-Ludwig Hausen;

McGraw-Hill, Inc. New York, NY, USA, (new version in progress) c.f.: http://portal.acm.org/citation.cfm?coll=GUIDE\&dl=GUIDE\&id=561101

2 A method for software evaluation; Dieter Welzel, Hans-Ludwig Hausen; Computer Standards \& Interfaces; Volume 17 , Issue 1, Pages: 121 - 129; c.f.: http://portal.acm.org/citation.cfm?id=198664

3 Guides to Software Evaluation for Acceptance Testing; Hans-Ludwig Hausen, Internal Workbook, (available from the author) 